\title{
Update Entlassmanagement
}

\section{Vertragspartner einigen sich auf Änderungen des Rahmenvertrags}

Auf in die nächste (und vielleicht letzte) Runde zum Thema Entlassmanagement! Letzter Stand war die Klage der Deutschen Krankenhausgesellschaft (DKG) gegen die Schiedsamtsentscheidung zum Rahmenvertrag, der als „letzter" Schritt zur Umsetzung erforderlich ist (siehe auch [1]).

\section{KANR statt LANR}

Nunmehr hat die DKG angekündigt, ihre Klage beim Landessozialgericht BerlinBrandenburg gegen die Schiedsamtsentscheidung zurückzuziehen, falls ein paar gesetzliche Änderungen vorgenommen werden.

Wesentlicher Punkt hierbei ist die von Beginn an im Streit stehende lebenslange Arztnummer für Krankenhausärzte. In der bisherigen Fassung des Rahmenvertrags (§ 6 Abs. 5 in der Fassung, die durch das Bundesschiedsamt festgesetzt wurde) waren die Krankenhäuser zur Anwendung der von den Kassenärztlichen Vereinigungen vergebenen vertragsärztlichen Arztnummern (sogenannte lebenslange Arztnummer - LANR) verpflichtet worden. Diese Regelung stieß auf massive Kritik bei den Krankenhäusern. Nach Klageerhebung fanden offensichtlich Gespräche unter Einbindung des Bundesministeriums für

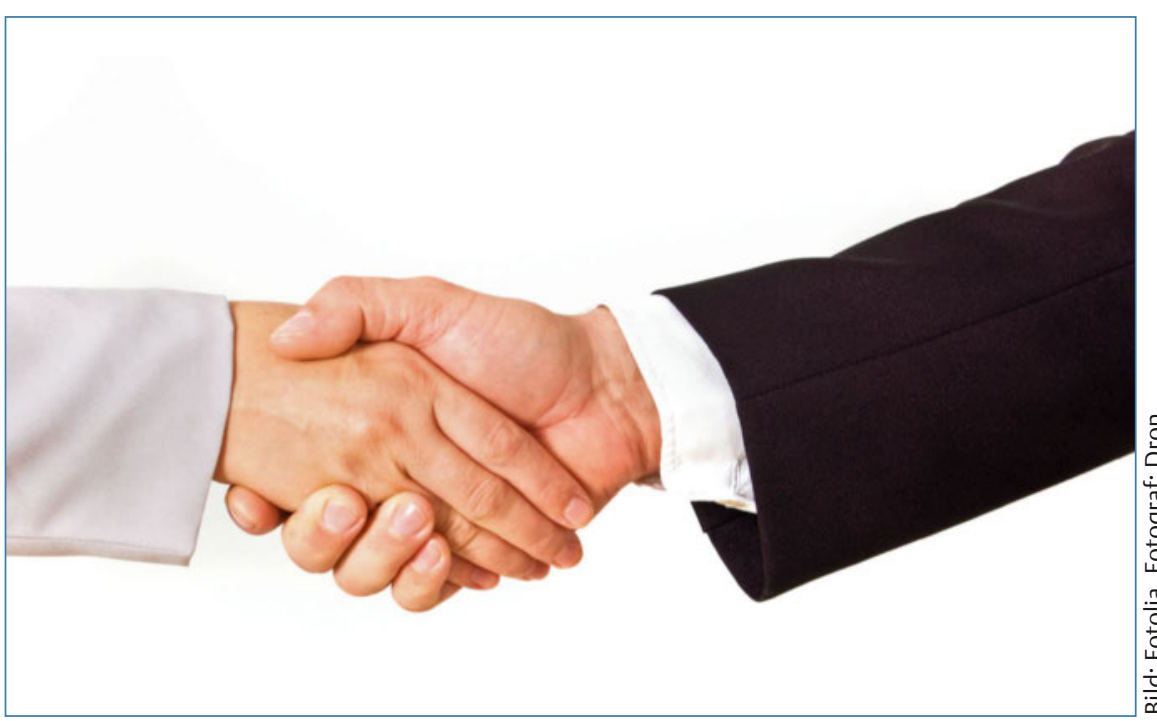

Gesundheit statt, die dazu führten, dass die Koalition einen Änderungsantrag in die Beratungen des Blut- und Gewebegesetzes (quasi als zeitlich nächstes gesetzliches Vehikel zur Änderung der gesetzlichen Regelungen im SGB V für das Entlassmanagement) einreichte. Inhalt des Änderungsantrags ist, dass in $\S 39$ Abs. 1 a SGB V zunächst klargestellt wird, dass die LANR im Entlassmanagement nicht angewendet wird. Krankenhausärzte müssten sich dann doch nicht bei den Kassenärztlichen Vereinigungen registrieren lassen. Ausreichend soll vielmehr eine - noch zu schaffende sogenannte Krankenhausarztnummer (KANR) für alle Krankenhausärzte, die in einem von der DKG und dem GKV-Spitzenverband bis zum 31.12.2018 zu erstellenden, bundesweiten Verzeichnis aufgeführt sind, sein. Die Rahmenvertragsparteien sollen gesetzlich dazu verpflichtet werden, bis zu diesem Zeitpunkt die Verwendung eines alternativen Kennzeichens zu vereinbaren. Geplant ist, dass die Krankenhäuser als „Übergangsregelung“ auf den Verordnungsmustern eine 9-stellige Fachgruppennummer angeben. Einzelheiten dazu finden sich sowohl im aktuell vorliegenden Rahmenvertragsentwurf als auch in den sogenannten Umsetzungshinweisen (s.u.).

Neben der Abschaffung der LANR für Krankenhausärzte wurden noch ein paar
Änderungen im Rahmenvertrag verhandelt, die ebenfalls umgesetzt werden sollen:

\section{Stationsäquivalente Behandlung}

Der Anwendungsbereich soll von Patienten aus voll- und teilstationären auf stationsäquivalente Behandlungen durch das Krankenhaus erweitert werden.

\section{Dokumentation}

Darüber hinaus ist eine ergänzende Regelung für den Fall vorgesehen, dass im Assessment festgestellt wird, dass der Patient keiner Anschlussversorgung bedarf. Dies wäre dann in der Patientenakte zu dokumentieren. In diesen Fällen soll dann keine weitere Einwilligung nach Anlage 1 b erforderlich sein.

\section{EM-Rezepte nur durch Fachärzte}

Änderungen sind auch im Hinblick auf die Verordnungsleistungen vorgesehen. Hier wurde ein Zusatz verhandelt, wonach das Verordnungsrecht durch Krankenhausärzte nur mit abgeschlossener Facharztweiterbildung ausgeübt werden kann.

\section{Voraussichtlicher Start 01.10 .2017}

Die Vertragspartner haben sich darauf verständigt, das Inkrafttreten des Rahmenvertrages auf den 01.10.2017 zu verschieben - vorbehaltlich der gesetzlichen Änderung insbesondere zur LANR. Und so ist der Ablauf geplant:

Die Änderungsvereinbarungen des Rahmenvertrags durch die Vertragsparteien liegen bereits vor. Die 2./3. Lesung des Blut- und Gewebegesetzes (mit dem entsprechenden Änderungsantrag zum 
Entlassmanagement) soll Anfang Juni 2017 im Bundestag erfolgen. Nach Verabschiedung der entsprechenden gesetzlichen Regelungen soll die Unterzeichnung der geänderten Rahmenvereinbarung stattfinden. Nach der Unterschrift des geänderten Rahmenvertrages würde die DKG die Klage gegen den geschiedsten Rahmenvertrag zurücknehmen.

Die KBV hatte darüber hinaus einen Antrag auf Sofortvollzug des Rahmenvertrags gestellt. Dieser Antrag ruht aktuell. Diesen Antrag würde die KBV nach Unterzeichnung des geänderten Rahmenvertrages zurücknehmen.

\section{Umsetzungshinweise der DKG}

Damit die Krankenhäuser bereits mit den umfangreichen Vorarbeiten zum Start des Entlassmanagements beginnen können, hat die DKG Umsetzungshinweise (im Mai 2017) zum Rahmenvertrag erarbeitet. Diese sollen offensichtlich dynamisch bei Bedarf angepasst werden (zu finden unter www.dkgev.de).

Die Umsetzungshinweise betreffen 4 Bereiche:

\section{Grundsätzliches zum Rahmenvertrag}

In den Umsetzungshinweisen finden sich zum einen die von den Vertragspartnern abgestimmten, gewünschten Änderungen. Darüber hinaus finden sich zu jeder Regelung (soweit erforderlich) ausführliche Hinweise zum Inhalt der Regelung und wertvolle „Hinweise aus Sicht der IT“.

\section{Anlage 1: Hinweise zum Verordnungswesen im EM}

Dies ist eine Pflichtlektüre für jedes Krankenhaus, das sich mit dem Gedanken trägt, Verordnungen im Entlassmanagement auszustellen. Nach dem Studium dieser 36 (!) Seiten ist dem Leser zumindest deutlich vor Augen geführt worden, auf welchem „Minenfeld“ man sich dabei bewegt.

\section{Anlage 2: Hinweise zu einem möglichen Assessmentbogen}

Hier werden mögliche Inhalte eines Assessmentbogens aufgezeigt, die freilich an die konkreten Gegebenheiten des einzelnen Krankenhauses angepasst werden müssen.

\section{Anlage 3: Checkliste Entlassplan}

Auf 7 Seiten werden die Mindestinhalte des vorgeschriebenen Entlassplans zusammengestellt.

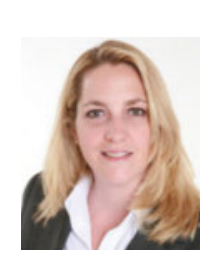

Dr. iur. Isabel Häser

Rechtsanwältin

Fachanwältin für Medizinrecht

Haimhauser Str. 1

80802 München

haeser@kanzlei-haeser.de

\section{Fazit}

Die DKG bemüht sich nach Kräften, den Krankenhäusern mit ihren Umsetzungshinweisen die Vorbereitung zum Start des Entlassmanagements einfacher zu gestalten. Jedoch sieht man alleine am Erläuterungsumfang, der im Übrigen noch zahlreiche Interpretationen enthält, wie komplex die Umsetzung der Materie in der Praxis tatsächlich ist.

Ob der Fahrplan mit Start am 01.10.2017 wirklich eingehalten werden kann, hängt nun davon ab, ob die gesetzlichen Änderungen so durchgehen, wie von den Vertragsparteien gewünscht. Aufgrund der mittlerweile vorliegenden Umsetzungshinweise wird ein Abwarten der Krankenhäuser wohl nicht mehr sinnvoll sein. Vielmehr sollte jedes Krankenhaus die Bereiche, die bereits vorbereitet werden können, angehen (z.B. Assessments festlegen). Insoweit ist eine Überprüfung der Abläufe und ein Standardisieren der Prozesse im Rahmen des Entlassmanagements (im vernünftigen Rahmen) zumindest nicht schädlich, selbst, wenn die Regelungen noch nicht Kraft treten sollten. Soweit vom Verordnungsrecht Gebrauch gemacht werden soll, ist es dringend erforderlich, die zukünftig verordnenden Ärzte auf die Verordnungsbedingungen im ambulanten Bereich zu schulen, um ein böses (und vor allem teures) Erwachen zu verhindern. Immerhin könnte sich durch den „Facharztvorbehalt“ die Anzahl der zu schulenden Krankenhausärzte etwas minimieren.

\section{Literatur}

[1] Häser I. Entlassmanagement - DKG klagt gegen Schiedsspruch. Klinikarz 2017; 46: 10-12 